Participatory Educational Research (PER)

Special Issue 2016-II, pp., 157-172; 01 February 2016

Available online at http://www.partedres.com

ISSN: $2148-6123$

http://dx.doi.org/10.17275/per.16.spi.2.15

\title{
Primary School Teachers' Perceptions of School Report
}

\author{
Nida BAYINDIR*, Levent SEVİ and Aynur ÇUKURCALIOĞLU \\ Dumlupinar University, Kütahya, Turkey
}

\begin{abstract}
School report is a document prepared by a teacher to follow up and evaluate a student's progress in lessons throughout a certain educational period. It is also an academic tool that allows the student and parents to be informed about the progress during that time and to see the shortcomings and to take the necessary precautions. The main objective of school report is not to show students' scores or to determine whether they have passed a lesson, but rather, to give information about their current state, to manage their skills by taking their differences into account, to guide them according to their interests, needs and skills, to determine the difficulties and deficiencies they come up against throughout the learning process and to help do away with them. However, if a school report is only considered in terms of scores and so becomes an indicator of students' success or failure, taking the report as a reference, the students whose report shows them successful might consider themselves more accepted, more important and valuable. Those with lower academic success, on the other hand, are naturally driven into a defensive psychology, which causes serious problems because the report has become the main target and is only assessed in terms of scores. The fault of parents and teachers to regard school report as an aim to achieve affects students negatively in many ways and might cause psychological problems in them in the short term. Perspectives arising from wrong responses, worries and fears, comparisons, high expectations, attributing failure to personality, critics without thinking, weathering and unnecessary rewards or punishments once again reveal the importance of school report worry. The research aims to reveal how primary school teachers, the first step of teaching, perceive school report. Teacher's perception of school report is the variable playing a fundamental role in shaping both student's and parents' definition of and expectations from school report. The research encompasses 190 primary school teachers in the city of Kütahya. According to the research results, $62,6 \%$ of the teachers think that school report shows the lessons the students are good at and 61,6\% think that school report shows the lessons the students are deficient in and their academic progress while 59,5\% consider it only as a tool. The teachers don't consider school report as a means of punishment and as the determiner of personality, and they think that it shouldn't be a source of honor or shame. The findings show that primary school teachers' perceptions of school report are mostly positive in favor of students. In this respect, it can be said that they perceive school report relevantly to its purpose. Regardless of the age group of the students, not turning the repot period into a trauma is based upon not only teachers' but also parents and students' perception of school report. Primary school teachers should reflect their perceptions of school report to students and parents and should also tell them that school reports are only a route map involving certain indicators in the early period and that all students are precious regardless of their reports. As regards to the school aspect, counsellors should explain the purpose of school report to teachers,
\end{abstract}

\footnotetext{
* Correspondence: , nidabayindir@yahoo.com
} 
parents and students and should provide the required guidance and warnings. When media is considered, the traditional school report understanding should be evaded and by sensitively planning the stress factors of school report like report festivals, awards and ads, it should be reiterated that school report is a documentation of students' mean scores. With the cooperation between the Directorates of National Education and Education Faculties, preservice teachers should be provided such training during theoretical and practical applications of assessment and evaluation lessons that shows school report as a tool depending on a process, uses scientific assessment and evaluation arguments, and gives priority to student psychology and health. Perceptions of students and parents about school report should be researched. School reports that don't only provide quantitative information but also give student success and success areas prominence should be designed; that is, as well as academic scores, school reports should also involve student success, areas requiring progress, parents' roles in teaching process, what students will/should do at school, statistical graphs showing previous success levels, in-class success, expected success levels, thoughts of the teacher, student and parents, study habits, target and suggestions.

Key words: Primary school teachers, School report, Perception of school report, Assessment and evaluation

\section{Introduction}

One of the most important indicators showing to what extent education programs and teaching-learning activities at school are successful is evaluation and assessment results (Özdemir, 2010, p. 788). Determining whether the behaviors expected to be gained by students at the end of education process are acquired by students or to what extent they are acquired is possible through assessment. As an inseparable and integral element of education, assessment is based on evaluations conducted at the beginning, during and at the end of education process (Çoklar, Vural and Şahin, 2009, p. 36, Gök \& Şahin, 2009, p. 130). Seeing the marks showing their performance and success throughout a period also encourages the students.

For a qualitative education, the changes in the development of students should be evaluated objectively. The objective in evaluation should be determining the access level of students to the expected gains and thus being able to get the necessary precautions timely. Therefore, the teacher achieves providing unbiased and accurate feedback through his/her objective assessment (Acar \& An11, 2009, p. 355). It is supposed to know whether the education process is successful or not. Early diagnosis of failure and failing individuals while the education process continues facilitates taking the required precautions. Knowing the success level and revealing the failure cases help to plan the similar education activities in the future on more realistic bases (Özdemir, 2010, p. 788).

Evaluation methods used for various purposes are involved in the education environment efficiently and prevalently. The most basic objective of using these methods in the education environment is to bring out student success effectively. While doing this, not one but various methods are used (Çoklar et al., 2009, p. 36). Unlike the traditional evaluation and assessment methods, the new ones in the elementary teaching program are such that monitor student development and regard assessment as a part of learning. During assessment, instead of easily-learned information independent of one another depending on memorizing, meaningful interdependent deep information prevails. The new methods focus on scientific understanding and logic. The purpose of assessment is to not find out what the student hasn't learned but to reveal what s/he has understood. At this point, the teacher is not the only assessor because the 
student also participates in the assessment. Above all, the program stipulates using the new evaluation methods along with the traditional ones encouragingly (Acar \& An1l, 2009, p. 355356).

The structure of evaluation and assessment is mostly determined upon education system philosophy. The basis of the basic philosophy of the 1998 program, the behaviorist approach, is that learners can turn what they have learned into behaviors. 2004 programs are mostly based on constructivism philosophy, according to which a learner constructs the information in his/her mind upon previous experience. Therefore, it is natural that students perceive the same information differently due to their own experience (Kabapınar \& Ataman, 2010, p. 777). By force of this approach, instead of teacher-centered teaching, student-centered teaching is adopted; it is aimed to integrate the family and environment into student-teacher and student-student interaction (Gelbal \& Kelecioğlu, 2007, p. 136). The teacher is no longer the one who transfers the information to the student directly but the one who guides the learner (Anil \& Acar, 2008, p. 45). In the constructivist approach, evaluation and assessment is a part of the teaching process; functioning not only at the beginning and end of learning but rather, at every important point in the learning process. Compared with the previous approach, more and various evaluation instruments and methods are required. Along with the traditionally used pencil-paper tests, it is possible to assess student performance in every aspect at a wider angle by monitoring student behavior in and out of the class, evaluating his/her interest and attitude and thus involving him/her into the evaluation and assessment process (Gelbal \& Kelecioğlu, 2007, p. 136). Thanks to the new program, alternative evaluation and assessment methods and techniques entered the education life. For example, project stands out as one of the evaluation and assessment types (Kabapınar \& Ataman, 2010, p. 778).

Alternative assessments are defined as student-centered, meaningful, interesting and up-tostudent assessment strategies, beyond the traditional understanding. Compared with the subjective-type question techniques, it is seen that the alternative assessment involves efficient participation and high level thinking. While multiple-choice and true-false questions serve assessing the knowledge that students can memorize, alternative assessment, with a more realistic perspective, determined what students have been able to understand and do. Thus, alternative assessment is complementary for students with different learning styles and contributes to choosing the appropriate assessment type for each student. It is also stated that using different evaluation and assessment strategies in class can not only provide the best way to evaluate various characteristics of students but also raise success motivation and provides more thinking skills, thus increasing academic performance (Duban \& Küçükyılmaz, 2008, p. 771, Kuran \& Kanatl1, 2009, p. 212). At the same time, using multiple evaluation and assessment techniques keeps the student alert and ready in class.

Education is a matter of teamwork and therefore, it is the responsibility of all; not only schools. Family, school, National Education Directorate and the society should all work together in this team. Determining the reasons for failure in students is important in that all the stakeholders in education should provide data for policy makers and enforcers to guide them in their policies (Altun, 2009, p. 568). Interfamilial harmony, supportive parental attitudes and versatility in parent participation into school activities all have important effects on school success. The most important factor separating the failing students with low academic success from the others is that they are deprived of parental support and interest. Parental austerity, inconsistency and conflict are among the other risk factors in lower school success. School success of the students with supportive parents in terms of education is pretty 
high (Çelenk, 2003, p. 28). A solid coherence in school-parents-student triangle not only affects the psychological and social life of the student positively but also boosts school success. According to the results of a study by Yanpar (1992), primary school teachers have difficulties in evaluating student success and assessing the results and need in-service training on evaluation and assessment (Çakan, 2009, p.100).

School reports are the traditional way of informing parents about students' school success (Çelenk, 2003, p. 31). The most important outcome expected from a student is academic success (Çil, http://www.kariyerpenceresi.com). Another way of informing parents about students' school performance is filing the students' work samples at school. These files should include students' work samples assessed by the teacher, teacher's opinions about students' reading activities and students' writing samples (Çelenk, 2003, p. 32).

In order to determine the perceptions and deficiencies in this area and take the necessary precautions, teachers' perceptions about school report should be determined. This research is important in revealing the perception of school report which is the longitudinal indicator of student progress and letting school report be regarded as an evaluation and assessment instrument as it is really worth.

\section{Method}

The sampling of this descriptive research, aiming to determine how primary school teachers perceive school report, was composed of primary school teachers working at the state schools in the city of Kütahya during 2015-2016 education period. The sampling was decided upon ease of access. The research encompassed total 190 primary school teachers teaching 1st-2nd-3rd classes. The data were gathered through a one dimensional questionnaire of 50 items developed by the researcher. Cronbach Alpha reliability coefficient was found 0,886 and factor analysis was conducted to determine validity as seen in Table 1 below. While data interpretation, items above $50 \%$ were taken. The research is limited to the questionnaire items and the applied statistical methods.

\section{Findings} below.

The table for the perception of primary school teachers about school report is given

Tablo 1. Reliability Statistics

\begin{tabular}{|c|c|c|c|c|c|c|c|}
\hline \multicolumn{4}{|c|}{ Cronbach's Alpha } & \multicolumn{4}{|c|}{$\mathrm{N}$ of Items } \\
\hline & & & & 86 & & & 190 \\
\hline \multicolumn{8}{|c|}{ Summary Item Statistics } \\
\hline & Mean & Minimum & Maximum & Range & $\begin{array}{l}\text { Maximum / } \\
\text { Minimum }\end{array}$ & Variance & $\mathrm{N}$ of Items \\
\hline Item Means & 2,908 & 2,158 & 4,011 & 1,8853 & 1,859 & 0,256 & 50 \\
\hline \multicolumn{8}{|c|}{ KMO and Bartlett's Test } \\
\hline \multicolumn{4}{|c|}{$\begin{array}{ll}\text { Kaiser-Meyer-Olkin Measure of Sampling Adequacy. } \\
\text { Bartlett's Test of Sphericity } & \text { Approx. Chi-Square } \\
& \text { Df } \\
& \text { Sig. } \\
\end{array}$} & & & & $\begin{array}{r}, 791 \\
4223,549 \\
1225 \\
, 000\end{array}$ \\
\hline
\end{tabular}

Scale Statistics

\begin{tabular}{|c|c|c|c|}
\hline Mean & Variance & Std. Deviation & N of Items \\
\hline 145,4000 & 367,945 & 19,18189 & 50 \\
\hline
\end{tabular}


Table 2. It is just an indicator assessing academic works

\begin{tabular}{lrrrr} 
& Fr & \multicolumn{1}{c}{$\%$} & $\overline{\mathbf{X}}$ & SS \\
\hline Totally agree & 24 & 12,6 & 2,8474 & 1,19207 \\
\hline Agree & 70 & 36,8 & & \\
\hline Undecided & 16 & 8,4 & & \\
\hline Disagree & 71 & 37,4 & & \\
\hline Totally disagree & 9 & 4,7 & \\
\hline Total & 190 & 100,0 & \\
\hline
\end{tabular}

Table 3. It shows what the student is deficient in

\begin{tabular}{lrrrr}
\hline & Fr & \multicolumn{1}{c}{$\%$} & $\overline{\mathbf{X}}$ & \multicolumn{1}{c}{ SS } \\
\hline Totally agree & 22 & 11,6 & 2,3368 &, 92708 \\
\hline Agree & 117 & $\mathbf{6 1 , 6}$ & & \\
\hline Undecided & 19 & 10,0 & & \\
\hline Disagree & 29 & 15,3 & & \\
\hline Totally disagree & 3 & 1,6 & & \\
\hline Total & 190 & 100,0 & & \\
\hline
\end{tabular}

$61,6 \%$ of the participant teachers think that school report shows what the student is deficient in.

Table 4. It shows the lessons student is good at

\begin{tabular}{lrrrr}
\hline & Fr & \multicolumn{1}{c}{$\%$} & $\bar{X}$ & SS \\
\hline Totally agree & 23 & 12,1 & 2,3316 &, 94871 \\
\hline Agree & 119 & $\mathbf{6 2 , 6}$ & & \\
\hline Undecided & 13 & 6,8 & & \\
\hline Disagree & 32 & 16,8 & & \\
\hline Totally disagree & 3 & 1,6 & & \\
\hline Total & 190 & 100,0 & & \\
\hline
\end{tabular}

$62,6 \%$ of the participant teachers think that school report shows the lessons student is good at.

Table 5. It shows whether the student studies or not

\begin{tabular}{llrrrr}
\hline & \multicolumn{2}{l}{ Fr } & \multicolumn{1}{l}{$\%$} & $\bar{X}$ & \multicolumn{1}{c}{ SS } \\
\hline Totally agree & 25 & 13,2 & 2,5053 & 1,04272 \\
\cline { 2 - 6 } & Agree & 93 & 48,9 & & \\
\cline { 2 - 5 } & Undecided & 28 & 14,7 & & \\
\cline { 2 - 5 } & Disagree & 39 & 20,5 & & \\
\cline { 2 - 5 } & Totally disagree & 5 & 2,6 & \\
\cline { 2 - 5 } & Total & 190 & 100,0 & & \\
\hline
\end{tabular}

Table 6. It is a good feedback for parents

\begin{tabular}{llrrrr}
\hline & \multicolumn{1}{l}{ Fr } & \multicolumn{1}{l}{$\%$} & $\overline{\mathbf{X}}$ & \multicolumn{1}{l}{ SS } \\
\hline Totally agree & 25 & 13,2 & 2,3737 &, 95527 \\
\cline { 2 - 6 } & Agree & 104 & $\mathbf{5 4 , 7}$ & & \\
\cline { 2 - 6 } & Undecided & 30 & 15,8 & & \\
\cline { 2 - 6 } & Disagree & 27 & 14,2 & & \\
\cline { 2 - 6 } & Totally disagree & 4 & 2,1 & & \\
\cline { 2 - 5 } & Total & 190 & 100,0 & & \\
\hline
\end{tabular}

$54,7 \%$ of the participant teachers think that school report is a good feedback for parents.

Table 7. It is student's responsibility

\begin{tabular}{lrrrr} 
& \multicolumn{1}{c}{ Fr } & $\%$ & $\bar{X}$ & \multicolumn{1}{l}{ SS } \\
\hline Totally agree & 28 & 14,7 & 2,3632 &, 98653 \\
\hline Agree & 102 & $\mathbf{5 3 , 7}$ & & \\
\hline Undecided & 28 & 14,7 & & \\
\hline
\end{tabular}




\begin{tabular}{lrr}
\hline Disagree & 27 & 14,2 \\
\hline Totally disagree & 5 & 2,6 \\
\hline Total & 190 & 100,0 \\
\hline
\end{tabular}

$53,7 \%$ of the participant teachers consider school report student's responsibility.

Table 8. It is a roadmap showing the overall progress

\begin{tabular}{lrrrr} 
& \multicolumn{1}{c}{ Fr } & \multicolumn{1}{c}{$\%$} & $\overline{\mathbf{X}}$ & SS \\
\hline Totally agree & 20 & 10,5 & 2,3895 &, 94611 \\
\hline Agree & 112 & $\mathbf{5 8 , 9}$ & & \\
\hline Undecided & 28 & 14,7 & & \\
\hline Disagree & 24 & 12,6 & & \\
\hline $\begin{array}{l}\text { Totally } \\
\text { disagree }\end{array}$ & 6 & 3,2 & \\
\hline Total & 190 & 100,0 & \\
\hline
\end{tabular}

$58,9 \%$ of the participant teachers consider school report as a roadmap showing the overall progress.

Table 9. It shapes the future behaviors of the student

\begin{tabular}{lrrrc} 
& Fr & \multicolumn{1}{c}{$\%$} & $\bar{X}$ & \multicolumn{1}{c}{ SS } \\
\hline Totally agree & 21 & 11,1 & 2,6842 & 1,00542 \\
\hline Agree & 68 & 35,8 & & \\
\hline Undecided & 55 & 28,9 & & \\
\hline Disagree & 42 & 22,1 & & \\
\hline Totally disagree & 4 & 2,1 & & \\
\hline Total & 190 & 100,0 & &
\end{tabular}

Table 10. It gives the student insight

\begin{tabular}{llrrrr}
\hline & Fr & $\%$ & $\bar{X}$ & SS \\
\hline Totally agree & 7 & 3,7 & 2,6105 &, 83311 \\
\cline { 2 - 6 } & Agree & 92 & 48,4 & & \\
\cline { 2 - 5 } & Undecided & 63 & 33,2 & & \\
\cline { 2 - 5 } Disagree & 24 & 12,6 & & \\
\cline { 2 - 5 } Totally disagree & 4 & 2,1 & & \\
\cline { 2 - 5 } & Total & 190 & 100,0 & & \\
\hline
\end{tabular}

Table 11. It determines the final success of the student

\begin{tabular}{llrrrr}
\hline & Fr & $\%$ & $\bar{X}$ & SS \\
\hline Totally agree & 7 & 3,7 & 3,2053 & 1,10553 \\
\cline { 2 - 6 } Agree & 59 & 31,1 & & \\
\cline { 2 - 6 } Undecided & 32 & 16,8 & & \\
\cline { 2 - 5 } Disagree & 72 & 37,9 & & \\
\cline { 2 - 5 } Totally disagree & 20 & 10,5 & & \\
\cline { 2 - 5 } & Total & 190 & 100,0 & & \\
\hline
\end{tabular}

Table 12. It is a sum of scientific data

\begin{tabular}{llrrrr}
\hline & Fr & $\%$ & $\bar{X}$ & SS \\
\hline Totally agree & 12 & 6,3 & 2,8053 & 1,00210 \\
\cline { 2 - 6 } & Agree & 76 & 40,0 & & \\
\cline { 2 - 5 } & Undecided & 44 & 23,2 & & \\
\cline { 2 - 5 } Disagree & 53 & 27,9 & & \\
\cline { 2 - 5 } & Totally disagree & 5 & 2,6 & & \\
\cline { 2 - 5 } & Total & 190 & 100,0 & & \\
\hline
\end{tabular}

Table 13. It also shows the success of parents 


\begin{tabular}{llrrrr}
\hline & Fr & $\%$ & $\bar{X}$ & SS \\
\hline Totally agree & 24 & 12,6 & 2,4000 &, 95286 \\
\cline { 2 - 6 } & Agree & 103 & $\mathbf{5 4 , 2}$ & & \\
\cline { 2 - 5 } & Undecided & 28 & 14,7 & & \\
\cline { 2 - 5 } Disagree & 33 & 17,4 & & \\
\cline { 2 - 5 } Totally disagree & 2 & 1,1 & & \\
\cline { 2 - 5 } & 190 & 100,0 & & \\
\hline
\end{tabular}

$54,2 \%$ of the participant teachers think that school report also shows the success of parents.

Table 14. It is teacher's opinions

\begin{tabular}{llrrrl}
\hline & Fr & & \multicolumn{1}{c}{$\%$} & $\overline{\mathrm{X}}$ & \multicolumn{1}{c}{ SS } \\
\hline Totally agree & 16 & 8,4 & 2,5421 &, 98444 \\
\cline { 2 - 6 } & Agree & 103 & $\mathbf{5 4 , 2}$ & & \\
\cline { 2 - 6 } & Undecided & 27 & 14,2 & & \\
\cline { 2 - 6 } & Disagree & 40 & 21,1 & & \\
\cline { 2 - 5 } & Totally disagree & 4 & 2,1 & & \\
\cline { 2 - 5 } & Total & 190 & 100,0 & & \\
\hline
\end{tabular}

$54,2 \%$ of the participant teachers think that school report is teacher's opinions.

Table 15. It might cause fear

\begin{tabular}{llrrrr}
\hline & Fr & \multicolumn{1}{c}{$\%$} & $\bar{X}$ & \multicolumn{1}{c}{ SS } \\
\hline Totally agree & 11 & 5,8 & 2,8053 & 1,02300 \\
\cline { 2 - 6 } & Agree & 81 & 42,6 & & \\
\cline { 2 - 6 } & Undecided & 39 & 20,5 & & \\
\cline { 2 - 6 } Disagree & 52 & 27,4 & & \\
\cline { 2 - 6 } & Totally disagree & 7 & 3,7 & & \\
\cline { 2 - 5 } & Total & 190 & 100,0 & & \\
\hline
\end{tabular}

Table 16. It is a follow-up of student progress

\begin{tabular}{|c|c|c|c|c|}
\hline & Fr & $\%$ & $\bar{x}$ & SS \\
\hline Totally agree & 18 & 9,5 & 2,3000 & ,78982 \\
\hline Agree & 117 & 61,6 & & \\
\hline Undecided & 36 & 18,9 & & \\
\hline Disagree & 18 & 9,5 & & \\
\hline Totally disagree & 1 & ,5 & & \\
\hline Total & 190 & 100,0 & & \\
\hline
\end{tabular}

$61,6 \%$ of the participant teachers think that school report is a follow-up of student progress.

Table 17. It determines student's fields of interest

\begin{tabular}{|c|c|c|c|c|}
\hline & $\mathrm{Fr}$ & $\%$ & $\bar{x}$ & SS \\
\hline Totally agree & 12 & 6,3 & 2,8632 & 1,06020 \\
\hline Agree & 74 & 38,9 & & \\
\hline Undecided & 43 & 22,6 & & \\
\hline Disagree & 50 & 26,3 & & \\
\hline Totally disagree & 11 & 5,8 & & \\
\hline Total & 190 & 100,0 & & \\
\hline
\end{tabular}

Table 18. It shows student's deficiencies 


\begin{tabular}{lrrrr} 
& Fr & $\%$ & $\overline{\mathbf{X}}$ & SS \\
\hline Totally agree & 13 & 6,8 & 2,5105 &, 90700 \\
\hline Agree & 106 & $\mathbf{5 5 , 8}$ & & \\
\hline Undecided & 34 & 17,9 & & \\
\hline Disagree & 35 & 18,4 & & \\
\hline Totally disagree & 2 & 1,1 & & \\
\hline Total & 190 & 100,0 & & \\
\hline
\end{tabular}

$55,8 \%$ of the participant teachers think that school report shows student's deficiencies.

Table 19. It shows the real personality of the student

\begin{tabular}{llrrrl}
\hline & Fr & \multicolumn{1}{c}{$\%$} & $\bar{X}$ & \multicolumn{1}{c}{ SS } \\
\hline Totally agree & 1 &, 5 & 3,7526 &, 90669 \\
\cline { 2 - 6 } Agree & 23 & 12,1 & & \\
\cline { 2 - 6 } Undecided & 32 & 16,8 & & \\
\cline { 2 - 6 } Disagree & 100 & $\mathbf{5 2 , 6}$ & & \\
\cline { 2 - 6 } Totally disagree & 34 & 17,9 & & \\
\cline { 2 - 5 } & Total & 190 & 100,0 & & \\
\hline
\end{tabular}

$52,6 \%$ of the participant teachers don't think that school report shows the real personality of the student.

Table 20. It is an instrument for punishment

\begin{tabular}{lrrrr}
\hline & Fr & $\%$ & $\bar{X}$ & \multicolumn{1}{c}{ SS } \\
\hline Totally agree & 1 &, 5 & 4,0105 &, 76973 \\
\hline Agree & 9 & 4,7 & & \\
\cline { 2 - 6 } Undecided & 22 & 11,6 & & \\
\hline Disagree & 113 & $\mathbf{5 9 , 5}$ & & \\
\hline Totally disagree & 45 & 23,7 & & \\
\hline Total & 190 & 100,0 & & \\
\hline
\end{tabular}

$59,5 \%$ of the participant teachers don't think that school report is an instrument for punishment.

Table 21. It is an instrument for rewarding

\begin{tabular}{lrrrr}
\hline & Fr & $\%$ & $\bar{X}$ & SS \\
\hline Totally agree & 7 & 3,7 & 3,3632 & 1,10301 \\
\hline Agree & 49 & 25,8 & & \\
\cline { 2 - 5 } Undecided & 25 & 13,2 & & \\
\hline Disagree & 86 & 45,3 & & \\
\hline Totally disagree & 23 & 12,1 & & \\
\hline Total & 190 & 100,0 & & \\
\hline
\end{tabular}

Table 22. It is an instrument of enforcement

\begin{tabular}{lrrrc}
\hline & Fr & $\%$ & $\bar{X}$ & SS \\
\hline Totally agree & 6 & 3,2 & 3,3737 & 1,01957 \\
\hline Agree & 39 & 20,5 & & \\
\hline Undecided & 42 & 22,1 & & \\
\hline Disagree & 84 & 44,2 & & \\
\hline Totally disagree & 19 & 10,0 & & \\
\hline Total & 190 & 100,0 & & \\
\hline
\end{tabular}

Table 23. It is a turning point

\begin{tabular}{|c|c|c|c|c|}
\hline & $\mathrm{Fr}$ & $\%$ & $\overline{\mathrm{X}}$ & SS \\
\hline Totally agree & 2 & 1,1 & 3,7000 & ,83539 \\
\hline Agree & 15 & 7,9 & & \\
\hline
\end{tabular}




\begin{tabular}{lrr}
\hline Undecided & 46 & 24,2 \\
\hline Disagree & 102 & $\mathbf{5 3 , 7}$ \\
\hline Totally disagree & 25 & 13,2 \\
\hline Total & 190 & 100,0 \\
\hline
\end{tabular}

$53,7 \%$ of the participant teachers don't think that school report is a turning point.

Table 24. It should be a source of pride or shame

\begin{tabular}{lrrrrl}
\hline & Fr & \multicolumn{1}{c}{$\%$} & $\bar{X}$ & SS \\
\hline Totally agree & 2 & 1,1 & 3,6263 &, 96628 \\
\cline { 2 - 6 } & Agree & 31 & 16,3 & & \\
\cline { 2 - 6 } & Undecided & 32 & 16,8 & & \\
\cline { 2 - 6 } Disagree & 96 & $\mathbf{5 0 , 5}$ & & \\
\cline { 2 - 6 } & 29 & 15,3 & & \\
\cline { 2 - 5 } & Totally disagree & 190 & 100,0 & & \\
\hline
\end{tabular}

$50,5 \%$ of the participant teachers don't think that school report should be a source of pride or shame.

Table 25. It is the goal

\begin{tabular}{lrrrc}
\hline & Fr & \multicolumn{1}{c}{$\%$} & $\bar{X}$ & SS \\
\hline Totally agree & 8 & 4,2 & 3,4842 & 1,09702 \\
\hline Agree & 38 & 20,0 & & \\
\hline Undecided & 26 & 13,7 & & \\
\hline Disagree & 90 & 47,4 & & \\
\hline Totally disagree & 28 & 14,7 & & \\
\hline Total & 190 & 100,0 & & \\
\hline
\end{tabular}

Table 26. It is an instrument

\begin{tabular}{|c|c|c|c|c|}
\hline & $\mathrm{Fr}$ & $\%$ & $\bar{x}$ & SS \\
\hline Totally agree & 24 & 12,6 & 2,3684 & ,99259 \\
\hline Agree & 113 & 59,5 & & \\
\hline Undecided & 18 & 9,5 & & \\
\hline Disagree & 29 & 15,3 & & \\
\hline Totally disagree & 6 & 3,2 & & \\
\hline Total & 190 & 100,0 & & \\
\hline
\end{tabular}

$59,5 \%$ of the participant teachers think that school report is an instrument.

Table 27. It assesses the student as a whole

\begin{tabular}{|c|c|c|c|c|}
\hline & $\mathrm{Fr}$ & $\%$ & $\bar{x}$ & SS \\
\hline Totally agree & 3 & 1,6 & 3,1421 & ,96818 \\
\hline Agree & 56 & 29,5 & & \\
\hline Undecided & 54 & 28,4 & & \\
\hline Disagree & 65 & 34,2 & & \\
\hline Totally disagree & 12 & 6,3 & & \\
\hline Total & 190 & 100,0 & & \\
\hline
\end{tabular}

Table 28. It shows not only the marks but also student's behaviors

\begin{tabular}{|c|c|c|c|c|}
\hline & $\mathrm{Fr}$ & $\%$ & $\bar{x}$ & SS \\
\hline Totally agree & 9 & 4,7 & 2,7421 & ,99831 \\
\hline Agree & 93 & 48,9 & & \\
\hline Undecided & 31 & 16,3 & & \\
\hline
\end{tabular}




\begin{tabular}{lrr}
\hline Disagree & 52 & 27,4 \\
\cline { 2 - 3 } Totally disagree & 5 & 2,6 \\
\cline { 2 - 3 } Total & 190 & 100,0 \\
\hline
\end{tabular}

Table 29. It determines student's future

\begin{tabular}{lrrrl}
\hline & Fr & \multicolumn{1}{c}{$\%$} & $\overline{\mathrm{X}}$ & \multicolumn{1}{c}{ SS } \\
\hline Totally agree & 7 & 3,7 & 3,4684 & 1,03205 \\
\hline Agree & 34 & 17,9 & & \\
\hline Undecided & 34 & 17,9 & & \\
\hline Disagree & 93 & 48,9 & & \\
\hline Totally disagree & 22 & 11,6 & & \\
\hline Total & 190 & 100,0 & & \\
\hline
\end{tabular}

Table 30. It is recorded

\begin{tabular}{llrrr}
\hline & Fr & \multicolumn{1}{c}{$\%$} & $\bar{X}$ & \multicolumn{1}{c}{ SS } \\
\hline Totally agree & 20 & 10,5 & 2,3474 &, 88812 \\
\hline Agree & 112 & $\mathbf{5 8 , 9}$ & & \\
\hline Undecided & 35 & 18,4 & & \\
\hline Disagree & 18 & 9,5 & & \\
\cline { 2 - 5 } Totally disagree & 5 & 2,6 & & \\
\hline Total & 190 & 100,0 & & \\
\hline
\end{tabular}

$59,9 \%$ of the participant teachers consider school report as an instrument for recording.

Table 31. Its scores are the real determiners

\begin{tabular}{llrrrl}
\hline & Fr & & $\%$ & $\bar{X}$ & SS \\
\hline Totally agree & 3 & 1,6 & 3,1947 &, 98613 \\
\cline { 2 - 6 } & Agree & 56 & 29,5 & & \\
\cline { 2 - 6 } & Undecided & 44 & 23,2 & & \\
\cline { 2 - 6 } Disagree & 75 & 39,5 & & \\
\cline { 2 - 6 } & Totally disagree & 12 & 6,3 & & \\
\cline { 2 - 5 } & Total & 190 & 100,0 & & \\
\hline
\end{tabular}

Table 32. Its scores might be given subjectively

\begin{tabular}{llrrrl}
\hline & Fr & & $\%$ & $\bar{X}$ & \multicolumn{1}{c}{ SS } \\
\hline Totally agree & 9 & 4,7 & 2,7474 &, 95362 \\
\cline { 2 - 6 } & Agree & 84 & 44,2 & & \\
\cline { 2 - 6 } & Undecided & 48 & 25,3 & & \\
\cline { 2 - 6 } & Disagree & 44 & 23,2 & & \\
\cline { 2 - 6 } & Totally disagree & 5 & 2,6 & & \\
\cline { 2 - 5 } & Total & 190 & 100,0 & & \\
\hline
\end{tabular}

Table 33. It affects student's mental health

\begin{tabular}{|c|c|c|c|c|}
\hline & $\mathrm{Fr}$ & $\%$ & $\bar{x}$ & SS \\
\hline Totally agree & 6 & 3,2 & 2,6158 & ,92282 \\
\hline Agree & 107 & 56,3 & & \\
\hline Undecided & 37 & 19,5 & & \\
\hline Disagree & 34 & 17,9 & & \\
\hline Totally disagree & 6 & 3,2 & & \\
\hline Total & 190 & 100,0 & & \\
\hline
\end{tabular}

$56,3 \%$ of the participant teachers think that school report affects student's mental health.

Table 34. It is an instrument for comparison

\begin{tabular}{|c|c|c|c|c|}
\hline & $\mathrm{Fr}$ & $\%$ & $\bar{x}$ & \\
\hline Totally agree & 11 & 5,8 & 2,7895 & 1,07787 \\
\hline
\end{tabular}




\begin{tabular}{llr}
\hline Agree & 91 & 47,9 \\
\cline { 2 - 3 } Undecided & 25 & 13,2 \\
\cline { 2 - 3 } Disagree & 53 & 27,9 \\
\cline { 2 - 3 } Totally disagree & 10 & 5,3 \\
\cline { 2 - 3 } Total & 190 & 100,0 \\
\hline
\end{tabular}

Table 35. It is a reason for criticism

\begin{tabular}{llrrrl}
\hline & Fr & \multicolumn{1}{c}{$\%$} & $\bar{X}$ & SS \\
\hline Totally agree & 8 & 4,2 & 2,9789 & 1,07869 \\
\cline { 2 - 6 } Agree & 76 & 40,0 & & \\
\cline { 2 - 5 } & Undecided & 30 & 15,8 & & \\
\cline { 2 - 5 } Disagree & 64 & 33,7 & & \\
\cline { 2 - 5 } Totally disagree & 12 & 6,3 & & \\
\cline { 2 - 5 } & Total & 190 & 100,0 & & \\
\hline
\end{tabular}

Table 36. It is kept as a memory

\begin{tabular}{llrrr}
\hline & Fr & \multicolumn{1}{c}{$\%$} & $\overline{\mathbf{X}}$ & \multicolumn{1}{c}{ SS } \\
\hline Totally agree & 21 & 11,1 & 2,4211 &, 93241 \\
\cline { 2 - 5 } Agree & 102 & $\mathbf{5 3 , 7}$ & & \\
\cline { 2 - 5 } Undecided & 37 & 19,5 & & \\
\cline { 2 - 5 } Disagree & 26 & 13,7 & & \\
\cline { 2 - 5 } Totally disagree & 4 & 2,1 & & \\
\cline { 2 - 5 } Total & 190 & 100,0 & \\
\hline
\end{tabular}

$53,7 \%$ of the participant teachers consider school report as an instrument for memory.

Table 37. It is an instrument of target

\begin{tabular}{llrrrc}
\hline & Fr & \multicolumn{1}{c}{$\%$} & $\bar{X}$ & SS \\
\hline Totally agree & 13 & 6,8 & 2,8526 & 1,04369 \\
\hline Agree & 69 & 36,3 & & \\
\hline Undecided & 52 & 27,4 & & \\
\hline Disagree & 45 & 23,7 & & \\
\hline Totally disagree & 11 & 5,8 & & \\
\hline Total & 190 & 100,0 & & \\
\hline
\end{tabular}

Table 38. It is an instrument for motivation

\begin{tabular}{lrrrr}
\hline & Fr & \multicolumn{1}{c}{$\%$} & $\overline{\mathbf{X}}$ & \multicolumn{1}{c}{ SS } \\
\hline Totally agree & 21 & 11,1 & 2,3211 &, 87110 \\
\hline Agree & 113 & $\mathbf{5 9 , 5}$ & & \\
\cline { 2 - 6 } Undecided & 34 & 17,9 & & \\
\hline Disagree & 18 & 9,5 & & \\
\hline \multirow{2}{*}{ Totally disagree } & 4 & 2,1 & & \\
& 190 & 100,0 & & \\
\hline
\end{tabular}

$59,5 \%$ of the participant teachers think that school report is an instrument for motivation.

Table 39. It should be assessed together with parents

\begin{tabular}{llrrrl}
\hline & Fr & \multicolumn{1}{c}{$\%$} & $\bar{X}$ & \multicolumn{1}{c}{ SS } \\
\hline Totally agree & 43 & 22,6 & 2,1579 &, 96845 \\
\cline { 2 - 6 } Agree & 103 & $\mathbf{5 4 , 2}$ & & \\
\cline { 2 - 6 } & 19 & 10,0 & & \\
\cline { 2 - 5 } & Undecided & 21 & 11,1 & & \\
\cline { 2 - 5 } & 4 & 2,1 & & \\
\cline { 2 - 5 } & Totally disagree & 190 & 100,0 & & \\
\hline
\end{tabular}

$54,2 \%$ of the participant teachers think that school report should be assessed together with parents.

Table 40. It is open for general criticism

$\begin{array}{llll}\text { Fr } & \% & \overline{\mathrm{X}} & \mathrm{SS}\end{array}$




\begin{tabular}{lrrrr}
\hline Totally agree & 15 & 7,9 & 2,7895 & 1,06304 \\
\hline Agree & 81 & 42,6 & & \\
\hline Undecided & 28 & 14,7 & \\
\hline Disagree & 61 & 32,1 & \\
\hline Totally disagree & 5 & 2,6 & \\
\hline Total & 190 & 100,0 & \\
\hline
\end{tabular}

Table 41. It should be open for general criticism

\begin{tabular}{|c|c|c|c|c|}
\hline & $\mathrm{Fr}$ & $\%$ & $\bar{x}$ & SS \\
\hline Totally agree & 14 & 7,4 & 3,1053 & 1,12192 \\
\hline Agree & 56 & 29,5 & & \\
\hline Undecided & 28 & 14,7 & & \\
\hline Disagree & 80 & 42,1 & & \\
\hline Totally disagree & 12 & 6,3 & & \\
\hline Total & 190 & 100,0 & & \\
\hline
\end{tabular}

Table 42. It leads to crime

\begin{tabular}{lrrrr}
\hline & Fr & $\%$ & $\bar{X}$ & SS \\
\hline Totally agree & 5 & 2,6 & 3,6684 &, 95427 \\
\hline Agree & 20 & 10,5 & & \\
\hline Undecided & 38 & 20,0 & & \\
\hline Disagree & 97 & 51,1 & & \\
\hline Totally disagree & 30 & 15,8 & & \\
\hline Total & 190 & 100,0 & & \\
\hline
\end{tabular}

Table 43. It causes personality problems

\begin{tabular}{|c|c|c|c|c|}
\hline & $\mathrm{Fr}$ & $\%$ & $\overline{\mathrm{x}}$ & SS \\
\hline Totally agree & 8 & 4,2 & 3,3105 & 1,00443 \\
\hline Agree & 34 & 17,9 & & \\
\hline Undecided & 56 & 29,5 & & \\
\hline Disagree & 75 & 39,5 & & \\
\hline Totally disagree & 17 & 8,9 & & \\
\hline Total & 190 & 100,0 & & \\
\hline
\end{tabular}

Table 44. It is a cause of jealousy among students

\begin{tabular}{|c|c|c|c|c|}
\hline & $\mathrm{Fr}$ & $\%$ & $\bar{x}$ & SS \\
\hline Totally agree & 16 & 8,4 & 2,7368 & 1,07113 \\
\hline Agree & 83 & 43,7 & & \\
\hline Undecided & 35 & 18,4 & & \\
\hline Disagree & 47 & 24,7 & & \\
\hline Totally disagree & 9 & 4,7 & & \\
\hline Total & 190 & 100,0 & & \\
\hline
\end{tabular}

Table 45. It is a result

\begin{tabular}{lrrrr}
\hline & Fr & \multicolumn{1}{c}{$\%$} & $\overline{\mathbf{X}}$ & \multicolumn{1}{c}{ SS } \\
\hline Totally agree & 17 & 8,9 & 2,8789 & 1,13206 \\
\cline { 2 - 5 } Agree & 70 & 36,8 & & \\
\hline Undecided & 35 & 18,4 & & \\
\hline Disagree & 55 & 28,9 & & \\
\hline Totally disagree & 13 & 6,8 & & \\
\hline Total & 190 & 100,0 & & \\
\hline
\end{tabular}

Table 46. It is an artificial indicator of success

\begin{tabular}{lrrrr}
\hline & Fr & $\%$ & $\bar{X}$ & SS \\
\hline Totally agree & 22 & 11,6 & 2,5263 & 1,08713 \\
\cline { 2 - 5 } Agree & 101 & $\mathbf{5 3 , 2}$ & & \\
\hline Undecided & 23 & 12,1 & & \\
\hline Disagree & 33 & 17,4 & & \\
\hline Totally disagree & 11 & 5,8 & & \\
\hline
\end{tabular}




\begin{tabular}{ccc}
\hline Total & 190 & 100,0 \\
\hline
\end{tabular}

$53,2 \%$ of the participant teachers perceive school report as an artificial indicator of success.

Table 47. It is a process

\begin{tabular}{ccccc}
\hline & Fr & $\%$ & $\overline{\mathrm{X}}$ & SS \\
\hline Totally agree & 24 & 12,6 & 2,4158 &, 98709 \\
\hline Agree & 103 & $\mathbf{5 4 , 2}$ & & \\
\hline Undecided & 28 & 14,7 & & \\
\hline Disagree & 30 & 15,8 & & \\
\hline Totally disagree & 5 & 2,6 & & \\
\hline Total & 190 & 100,0 & & \\
\hline
\end{tabular}

$54,2 \%$ of the participant teachers consider school report as a process.

Table 48. It is an instrument for threat and pressure

\begin{tabular}{|c|c|c|c|c|}
\hline & $\mathrm{Fr}$ & $\%$ & $\bar{x}$ & SS \\
\hline Totally agree & 7 & 3,7 & 3,5368 & 1,05219 \\
\hline Agree & 30 & 15,8 & & \\
\hline Undecided & 37 & 19,5 & & \\
\hline Disagree & 86 & 45,3 & & \\
\hline Totally disagree & 30 & 15,8 & & \\
\hline Total & 190 & 100,0 & & \\
\hline
\end{tabular}

Table 49. It means expensive toys

\begin{tabular}{|c|c|c|c|c|}
\hline & $\mathrm{Fr}$ & $\%$ & $\bar{x}$ & SS \\
\hline Totally agree & 5 & 2,6 & 3,7105 & 94022 \\
\hline Agree & 18 & 9,5 & & \\
\hline Undecided & 35 & 18,4 & & \\
\hline Disagree & 101 & 53,2 & & \\
\hline Totally disagree & 31 & 16,3 & & \\
\hline Total & 190 & 100,0 & & \\
\hline
\end{tabular}

$53,2 \%$ of the participant teachers don't match school report with expensive toys.

Table 50. Bad school record means studying during summer

\begin{tabular}{|c|c|c|c|c|}
\hline & $\mathrm{Fr}$ & $\%$ & $\overline{\mathrm{X}}$ & SS \\
\hline Totally agree & 18 & 9,5 & 3,8368 &, 80980 \\
\hline Agree & 26 & 13,7 & & \\
\hline Undecided & 115 & 60,5 & & \\
\hline Disagree & 31 & 16,3 & & \\
\hline Totally disagree & 190 & 100,0 & & \\
\hline
\end{tabular}

$60,5 \%$ of the teachers don't regard school report as a loss of summer holiday.

Table 51. It is an ego satisfaction

\begin{tabular}{|c|c|c|c|c|}
\hline & Fr & $\%$ & $\bar{x}$ & SS \\
\hline Totally agree & 5 & 2,6 & 3,5526 & 1,04140 \\
\hline Agree & 33 & 17,4 & & \\
\hline Undecided & 35 & 18,4 & & \\
\hline Disagree & 86 & 45,3 & & \\
\hline Totally disagree & 31 & 16,3 & & \\
\hline Total & 190 & 100,0 & & \\
\hline
\end{tabular}

According to the research findings, $62,6 \%$ of the primary school teachers thinks that school 
report shows the lessons the student is good at, 61,6\% think that it shows the lessons the student is deficient in and student's progress while 59,5\% regard it as just an instrument. The teachers don't regard school report as an instrument for punishment or as a personality determiner and they think that it shouldn't be a source of pride or shame.

\section{Conclusion \& Suggestions}

It is stated in the articles of Regulation Primary Education Institutions about evaluation and assessment that success of primary school students should be designed as very good-good-needs improvement considering their development levels. (MEB, 2014). According to the research findings, primary school teachers mostly see school report as an instrument showing student's deficiencies and progress and the lessons s/he is good at. According to primary school teachers, school report is by no means an instrument for punishment, enforcement or a turning point. The findings show that school report perceptions of primary school teachers are generally positive in favor of students. As a matter of fact, there are no assessments reduced to scores in the evaluation parameters in the regulation. In this respect, it can be said that primary school teachers perceive school report in accordance with its purpose. Regardless of the student's age group, it is up to the teachers, parents and students' perceptions of school report for a student not to go through a trauma during school report time. School report is an indication of academic success. It is neither the end of the road nor a turning point. Use of school report as an enforcement-pressure, its threatening the mental health and its preparing a ground for undesired results are pathologic result of great expectations burdened on it. On the other hand, school report perceived as just a transcript without creating exclusion can only be regarded as a motivational instrument. It is not correct for teachers, students and parents to take the school report scores as a reference and assess success upon a document. Rather, the student should be assessed as a whole, his/her progress should be monitored, his/her strong sides should be improved thanks to school report.

Primary school teachers should instill the correct school report perception into students and parents and should tell them that school report is a roadmap including certain indicators at an early stage and the student is more important than all.

In terms of school dimension, school counselors should inform all teachers, parents and students about the purpose of school report and give them the necessary guidance and warnings.

In terms of media dimension, the traditional school report perception should be avoided, it should be reiterated that school report is the transcript showing the mean scores a student has achieved longitudinally and the stress factors related with school report such as school report festivals, awards, advertisements, etc. should be planned sensitively.

With the cooperation of National Education Ministry and education faculties, prospective teachers should be provided with such a training about school report in the theoretical and practical dimensions of evaluation and assessment courses that show school report as an assessment instrument based on process, can make use of scientific evaluation and assessment arguments and give priority to student health and psychology.

Students and parents' perceptions about school report should be determined through research.

School report design should be such that features student's success and progress (not only numeric data). Along with academic scores, school report should also include places showing 
student's success, points needing progress, parents roles in the teaching process, things to be done at school, statistical graphs showing the success levels in previous years, in-class performance, expected success levels, opinions of the student, parents and the teacher, study habits, goals and suggestions.

\section{References}

Acar, M. \& Anıl, D. (2009). Sınıf Öğretmenlerinin Performans Değerlendirme Sürecindeki Değerlendirme Yöntemlerini Kullanabilme Yeterlikleri, Karşılaştıkları Sorunlar Ve Çözüm Önerileri [Proficiency of Primary School Teachers in Using Assessment Methods during Performance Assessment Process, Problems They Encounter and Suggestions for Solution]. TUBAV Journal of Science, Vol:2, No:3, 354-363.

Akbaba, Altun, S. (2009). İlköğretim Öğrencilerinin Akademik Başarısızlıklarına İlişkin Veli, Öğretmen Ve Öğrenci Görüşlerinin İncelenmesi [Analysis of Parents, Teacher and Student's Opinions about Academic Failure of Primary School Students]. Primary Education Online, 8(2), 567-586. [Online]: Http://Ilkogretim-Online.Org.Tr

Anıl, D. \& Acar, M. (2008). Sınıf Öğretmenlerinin Ölçme Değerlendirme Sürecinde Karşılaştıkları Sorunlara İlişkin Görüşleri [Opinions of Primary School Teachers about The Problems They Encounter during Evaluation and Assessment Process]. 100 ${ }^{\text {th }}$ Year University, Journal of Education Faculty. December 2008. Vol:V, No:II, 44-61. Http://Efdergi.Yyu.Edu.Tr

Çakan, M, (2004). Öğretmenlerin Ölçme-Değerlendirme Uygulamaları Ve Yeterlik Düzeyleri: İlk Ve Ortaögretim [Evaluation and Assessment Practices of Teachers and Their Proficiency Levels: Primary and Secondary Education]. Ankara University Journal of Education Faculty, 37(2), 99-114.

Çelenk, S. (2003). Okul Başarısının Ön Koşulu: Okul Aile Dayanışması [Prerequisite for School Success: School-Parents Cooperation]. Primary Education-Online 2 (2), 28-34. Http://Www.İlkogretim-Online.Org.Tr

Çi1, A. Ders Başarısı Mı, Sosyal Başarı Mı? Karnelerin Görünmez/Görünemez Yüzü [Course Success or Social Success? Unseen Face of School Reports]. Http://Www.Kariyerpenceresi.Com/?Rehberlik,111/Ders-Basarisi-Mi-Sosyal-BasariMi.Html Accessed on 8th October 2015

Çoklar, A. N., Vural, L. and Şahin, Y. L. (2009). Öğretmen Adaylarının Uygulayabilecekleri Ölçme-Değerlendirme Yaklaşımları İle Ölçme-Değerlendirme Amaçlı Teknoloji Kullanım Özyeterlikleri [Evaluation and Assessment Approaches Prospective Teachers Can Practice and Their Self-Efficacy in Using Technology for Evaluation and Assessment]. Adiyaman University Journal of Institute of Social Sciences, Year: 2, No: 3, S. 35-54.

Duban, N. \& Küçükyılmaz, E. A. (2008). Sınıf Öğretmeni Adaylarının Alternatif ÖlçmeDeğerlendirme Yöntem Ve Tekniklerinin Uygulama Okullarında Kullanımına İlişkin Görüşleri [Opinions of Prospective Primary School Teachers about the Use of Alternative Evaluation and Assessment Methods and Techniques in Practice Schools]. Primary Teaching Online, 7(3), 769-784. [Online]: Http://Illkogretim-Online.Org.Tr

Gelbal, S. \& Kelecioğlu, H. (2007). Öğretmenlerin Ölçme Ve Değerlendirme Yöntemleri Hakkındaki Yeterlik Algıları Ve Karşılaştıkları Sorunlar [Proficiency Perceptions of Teachers about Evaluation and assessment Methods and Problems They Encounter]. Hacettepe University Journal of Education 33: 135-145.

Gök, B, \& Şahin, E, A, (2009). İlköğretim 4 Ve 5. Sınıf Öğretmenlerinin Değerlendirme Araçlarını Çoklu Kullanımı Ve Yeterlik Düzeyleri $\left[4^{\text {th }}\right.$ and $5^{\text {th }}$ Classes' Primary School 
Teachers' Multiple Use of Assessment Instruments and Their Proficiency Levels]. Education and Science, 34,(153), 127-143.

Kabapınar, Y. \& Ataman, M. (2010). İlköğretim Sosyal Bilgiler (4-5. Sınıf) Programları'ndaki Ölçme Ve Değerlendirme Yöntemlerine İlişkin Öğretmen Görüşleri [Teachers' Opinions about Evaluation and Assessment Methods in Primary Education Social Studies (4th-5th Classes) Program]. Primary Education Online, 9(2), 776-791. [Online]: Http://illkogretim-Online.Org.Tr

Kuran, K, \& Kanatlı, F, (2009). Alternatif Ölçme Değerlendirme Teknikleri Konusunda Sınıf Öğretmenlerinin Görüşlerinin Değerlendirilmesi [Analysis of Primary School Teachers' Opinions about Alternative Evaluation and Assessment Techniques]. Mustafa Kemal University Journal of Institute of Social Sciences, 6(12), 209-234.

MEB, (2014). Milli Eğitim Bakanlığı Okul Öncesi Eğitim Ve İlköğretim Kurumları Yönetmeliği [Ministry of National Education's Regulation of Institutes of Pre-School and Primary Education]. Official Gazette, No, 29072.

Özdemir, S. M. (2010). İlköğretim Öğretmenlerinin Alternatif Ölçme Ve Değerlendirme Araçlarına İlişkin Yeterlikleri Ve Hizmet İçi Eğitim İhtiyaçları [Proficiency of Primary School Teachers in Alternative Evaluation and Assessment Instruments \& Their Inservice Training Needs]. Journal of Turkish Education Sciences, Fall 2010, 8(4), 787816. 